\section{Kompass}

Nutrition \& Dietetics

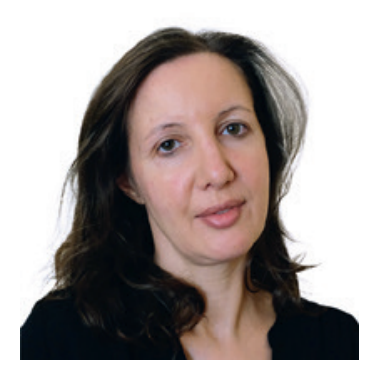

\title{
Determinants of Malnutrition in Older Adults
}

\author{
Cecilia Medeiros de Morais
}

Faculty of Nutrition and Food Sciences, University of Porto, Porto, Portugal

Abstract from Katsas K, Mamalaki E, Kontogianni MD, et al.: Malnutrition in older adults: Correlations with social, diet-related, and neuropsychological factors. Nutrition. 2020;71:110640.

\section{Keywords}

Cognitive status · Community-dwelling · Diet · Elderly ·

Malnutrition · Nutritional status

\begin{abstract}
Background: The number of older adults is increasing rapidly. Malnutrition is a major problem in this age group, which may adversely affect health and quality of life. Several physiological, socioeconomic, and neuropsychological factors can lead to malnutrition.
\end{abstract}

Objectives: The aim of this study was to evaluate the nutritional status of community-dwelling older adults, and explore the associations of malnutrition risk with physiological, socioeconomic, and neuropsychological characteristics.

Methods: This study is part of the Hellenic Longitudinal Investigation of Aging and Diet study, a cross-sectional observational study in Greece, and study participants were 1831 urban-dwelling elderly individuals (mean age: $73.1 \pm 5.9$ y; $40.8 \%$ men). Risk for malnutrition was assessed with the Determine Your Nutritional Health checklist. Data on age, sex, level of education, marital status, depression, cognitive performance, body mass index, total energy intake, and adherence to the Mediterranean diet were recorded. Correlations and multivariate analyses were performed between these variables and risk for malnutrition.

Results: The estimated prevalence of moderate and high nutritional risks was 34.8\% and 29.4\%, respectively. Risk for malnutrition was associated with marital status (unmarried), increased body mass index, male sex, lower level of education, lower cognitive performance, and lower adherence to the Mediterranean diet $(P<0.05)$.

Conclusions: Nutritional screening should be performed frequently in all community-dwelling older adults. Health experts should perform nutritional screening in all community-dwelling older adults as part of secondary prevention, and nutrition counselling and support should be offered in those at risk for malnutrition. 


\section{Knowledge Transfer}

\section{Background}

Older adults make up an increasingly larger group of the population in European countries. The scientific community recognises the need to promote healthy ageing and quality of life in older age. A number of studies have investigated the impact of several diet-related conditions in older adults, namely malnutrition and the factors associated with nutritional risk. In Greece, the "Hellenic Longitudinal Investigation in Ageing and Diet" study identified several factors related to the nutritional status of community-dwelling older adults, namely marital status, body mass index (BMI), sex, level of education, cognitive performance, and adherence to the Mediterranean diet. Also, there was a high prevalence of moderate and high nutritional risks, with only around one third of the participants being well-nourished. Nutritional screening in the community should be performed routinely, and older adults at risk should have support and nutritional counselling [1].

\section{Malnutrition in Older Adults}

Malnutrition is a state that results from a "lack of intake or uptake which can be caused by starvation, disease or advanced ageing (e.g. $>80$ years), alone or in combination" [2]. As a consequence, several physiological changes might occur such as loss of muscle mass, impaired immune function, decrease in bone mass, anaemia, poor healing, and cognitive impairment [1]. Malnutrition in older age brings about additional constraints as some of the body functions might already be compromised by the ageing process, resulting in poor prognosis in the case of disease and impaired clinical outcomes. The most common nutritional risk screening tools validated for older adults are the Mini Nutritional Assessment (MNA), the Malnutrition Universal Screening Tool (MUST), and the Nutritional Risk Screening 2002 (NRS). A prevalence of malnutrition of around 20\% was found in studies using the MNA, but there were differences between the settings (rehabilitation, 50.5\%; hospital, 38.7\%; nursing home, 13.8\%; community, 5.8\%) [3]. The Determine your Nutritional Health (DETERMINE) Checklist for screening has been used in community-dwelling settings [4]. Risk screening in the community is an essential prevention measure since it allows combatting malnutrition in the early stages and finding nutritional deficiencies before the health status of older adults declines [3].

\section{Determinants of Malnutrition in Older Age}

The Helenic Study found that older adults who were at higher risk of malnutrition were female, unmarried, presented a higher BMI, fewer years of education, lower global cognitive function (GCF), and a lower adherence to the Mediterranean diet • Other conditions increasing the risk of malnutrition were: use of medication (more than
3 drugs per day), an illness or condition that influenced food intake, dental or oral problems, eating few fruits and vegetables or milk products, and eating alone most of the time [1]. Previous research using the same screening tool found similar data, and participants also reported not always having enough money for food [5].

Presenting the factors by section, critical factors for malnutrition in older adults involve oral problems (dental status, difficulties with chewing or swallowing, oral pain, and gum issues), psychosocial circumstances (cognitive function, depression, psychological distress, anxiety, social support, living conditions, access to transport, loneliness, wellbeing), medication and access to healthcare, health (comorbidities, self-perceived health), physical function, and diet. Most of the factors were identified in one or more studies, nevertheless there is a lack of robust evidence for most of the determinants $[4,6]$.

\section{Conclusion}

Although the scientific evidence is not always strong concerning the factors associated with malnutrition, considering the various dimensions of malnutrition and potential causes, it is important to implement risk assessment procedures for older adults living in the community and thus enable proper follow-up of those at risk.

\section{Disclosure Statement}

I hereby declare that there are no conflicts of interest with regard to this commentary.

\section{References}

1 Katsas K, Mamalaki E, Kontogianni MD, et al.: Malnutrition in older adults: Correlations with social, diet-related, and neuropsychological factors. Nutrition. 2020;71:110640

2 Cederholm T, Barazzoni R, Austin P, et al.: ESPEN guidelines on definitions and terminology of clinical nutrition. Clin Nutr. 2017;36(1):49-64.

3 Kaiser MJ, Bauer JM, Rämsch C, et al.; Mini Nutritional Assessment International Group. Frequency of malnutrition in older adults: a multinational perspective using the mini nutritional assessment. J Am Geriatr Soc. 2010;58(9):17341738.

4 Corish CA, Bardon LA. Malnutrition in older adults: screening and determinants. Proc Nutr Soc. 2019;78(3):372-379.

5 de Morais C, Oliveira B, Afonso C, et al.: Nutritional risk of European elderly. Eur J Clin Nutr. 2013;67(11):1215-1219.

6 O'Keeffe M, Kelly M, O'Herlihy E, et al.: MaNuEL consortium. Potentially modifiable determinants of malnutrition in older adults: A systematic review. Clin Nutr. 2019;38(6):2477-2498.

Correspondence to:

Cecilia Medeiros de Morais, cecilia_mmorais@hotmail.com 